\begin{tabular}{|c|c|c|c|c|c|c|}
\hline \multicolumn{7}{|l|}{ Patient No } \\
\hline Duration (h) & Cerebral-rSO2 & c-FTOE & Abdominal-rSO2 & a-FTOE & Renal-rSO2 & r-FTOE \\
\hline $1(58)$ & $73 \pm 6,9$ & $0,2 \pm 0,07$ & $77 \pm 6,4$ & $0,2 \pm 0,03$ & $71 \pm 6,8$ & $0,2 \pm 0,05$ \\
\hline $2(68)$ & $78 \pm 3$ & $0,2 \pm 0,01$ & $77 \pm 5,5$ & $0,2 \pm 0,03$ & $78 \pm 5,2$ & $0,2 \pm 0,01$ \\
\hline 3 before surgery on PGE1 (112) & $57 \pm 6,8$ & $0,3 \pm 0,07$ & $63 \pm 8,5$ & $0,2 \pm 0,08$ & $72 \pm 7,2$ & $0,1 \pm 0,08$ \\
\hline 4 after surgery (25) & $64 \pm 3,6$ & $0,1 \pm 0,05$ & $68 \pm 0,6$ & $0,02 \pm 0,001$ & $61 \pm 3,2$ & $0,1 \pm 0,05$ \\
\hline
\end{tabular}

regarding not only cerebral but abdominal or renal tissue oxygenation (rSO2) as well. Fractional tissue oxygen extraction (FTOE) is calculated from NIRS measurements and arterial haemoglobin oxygen saturation (SpO2) measured by pulse oxymeter. Multichannel NIRS devices maybe very helpful in newborns with multisystem problems enabling realtime simultaneous measurements of $\mathrm{rSO} 2$ from different parts of the body. Pulse oxymeter integrated into a multichannel NIRS device provides simultaneous $\mathrm{SpO} 2$ monitoring making FTOE calculations more accurate and easier.

Methods Three term newborns; 2 undergoing therapeutic hypothermia for hypoxic ischaemic encephalopathy grade II, 1 with critical pulmonary stenosis before and after cardiac surgery were monitored by multichannel NIRS (Sensmart X-100, NONIN, USA) device including cerebral, abdominal, renal $\mathrm{rSO} 2$ and $\mathrm{SpO} 2$ probes. FTOE was calculated using the equation; (SpO2$\mathrm{rSO} 2) / \mathrm{SpO} 2$.

Results Duration of monitorization and $\mathrm{rSO} 2$ and FTOE values of different body sites are presented in table with mean \pm SD.

Discussion Longterm simultaneous monitoring of tissue oxygenation in brain, abdomen and kidneys is useful while following newborns with multisystem problems requiring hypothermia or circulatory medications to titrate the treatment accordingly. NIRS device with integrated pulse oxymeter maybe helpful for realtime calculation of FTOE to assess hemodynamics.

\section{PO-0495 INTRAVENOUS IBUPROFEN (IBU) VS CONTINUOUS INDOMETHACIN-INFUSION (IND-INF) FOR SYMPTOMATIC PATENT DUCTUS ARTERIOSUS (PDA) TREATMENT IN NEWBORNS}

N Storring, P Amess, N Aiton, R Bomont, H Rabe, JR Fernandez Alvarez. Neonatology, Brighton and Sussex University Hospitals NHS Trust, Brighton, UK

10.1136/archdischild-2014-307384.1141

\begin{tabular}{llll} 
Abstract PO-0495 Table 1 & & \\
\hline Outcome & IND-INF & IBU & p-value \\
cPDA(n/N) & $1 / 8$ & $2 / 8$ & 1 \\
rPDA(n/N) & $6 / 8$ & $3 / 8$ & 0.1 \\
wPDA(mm) & $1.5(1.3-1.7)$ & $1.5(1.1-1.5)$ & 1 \\
rePDA(n/N) & $0 / 8$ & $2 / 8$ & 0.15 \\
FiO $(\%)$ & $29(25-40)$ & $29(25-36)$ & 0.96 \\
$\mathrm{BPD}(\mathrm{n} / \mathrm{N})$ & $7 / 8$ & $8 / 8$ & 1 \\
$\mathrm{SOD}(\mathrm{mmol} / \mathrm{l})$ & $131(128-133)$ & $138(133-141)$ & 0.11 \\
$\mathrm{CREA}(\mathrm{mmol} / \mathrm{l})$ & $57(51-77)$ & $77(66-95)$ & 0.11 \\
IP(n/N) & $0 / 8$ & $1 / 8$ & 1 \\
$\mathrm{NEC}(\mathrm{n} / \mathrm{N})$ & $1 / 8$ & $0 / 8$ & 1 \\
THROM(x10 $/$ microliter $)$ & $288(162-407)$ & $281(175-330)$ & 0.66 \\
$\mathrm{cRI}(\mathrm{n} / \mathrm{N})$ & $0.9(0.8-0.9)$ & $0.75(0.65-0.85)$ & 0.11 \\
IVH(n/N) & $3 / 8$ & $2 / 8$ & 1 \\
$\mathrm{ROP}(\mathrm{n} / \mathrm{N})$ & $3 / 8$ & $2 / 8$ & 1 \\
$\mathrm{Mortality}(\mathrm{n} / \mathrm{N})$ & $0 / 8$ & $0 / 8$ & 1 \\
\hline
\end{tabular}

Background IBU is equivalent to IND for PDA-treatment. IBU reduces less cerebral, mesenteric and renal perfusion and platelet function. IND-INF seems to have similar effects on circulation, but the clinical efficacy of this approach is unclear.

Aim To compare the efficacy and safety of PDA-treatment using 36-hour IND-INF vs IBU in preterm infants.

Methods Retrospective matched-pair cohort-analysis of infants $<28$ GA from a tertiary centre (2012-2014). Infants matched for: Chorioamnionitis, antenatal steroids, vaginal delivery, GA, birthweight, gender, surfactant-administration, mechanical-ventilation, oxygen-requirement (FiO2), PDA-width (wPDA), inotropes, fluid-intake, plasma sodium (SOD), plasma-creatinine (CREA), platelet count (THROM), cerebral resistance-index (cRI) and treatment-age. Outcome measures: wPDA, number of closed (cPDA), restrictive (rPDA), re-opened (rePDA) PDA; FiO2, BPD, SOD, CREA, intestinal perforation (IP), necrotising enterocolitis (NEC), THROM, cRI, IVH, ROP, mortality. Data-presentation: Median (interquartile range) or ratio $(\mathrm{n} / \mathrm{N})$. Data-analysis: Fisher's-Exact-/Mann-Whitney-Test $(\mathrm{p}<0.05)$.

Results 16 newborns (8 IND-INF/8 IBU) recruited. Baselinecharacteristics (GA 26 [25-27] vs 26 [25-26], p = 0.65; birthweight $842 \mathrm{~g}$ [597-925] vs $777 \mathrm{~g}$ [730-801], $\mathrm{p}=0.88$; rest not displayed) and outcomes were not significantly different:

Conclusion IND-INF appears to be as safe as IBU whilst maintaining the same efficacy for treatment of symptomatic PDA in newborns $<28$ GA.

\section{PO-0496 CENTRAL VENOUS CATHETER RELATED THROMBUS ON THE NEONATAL UNIT: PRESENTATION, CLINICAL COURSE AND MANAGEMENT}

R Pramod, $\underline{K}$ Johnson, SJ English. Neonatal Medicine D Floor Martin Wing, Leeds Teaching Hospital NHS Trust, Leeds West Yorkshire, UK

\subsection{6/archdischild-2014-307384.1142}

Background and aims The placement of a Central Venous Catheter (CVC) is routine clinical practice for sick and preterm infants on the Neonatal Intensive Care Unit (NICU). Such catheters are vital to ensure reliable and continuous delivery of nutrition and medication.

Thrombus related to the placement of such catheters is well described. ${ }^{1}$

Abstract PO-0496 Table 1 Gestation and site of thrombus

\begin{tabular}{ll}
\hline Gestation & Site \\
$32+1$ & IVC, portal vein. \\
$27+1$ & IVC, portal, renal veins. \\
$27+5$ & IVC, renal veins. \\
$36+5$ & Portal vein. \\
$24+1$ & IVC, renal vein. \\
$24+4$ & IVC \\
$25+5$ & IVC, common iliac, renal vein.
\end{tabular}

